\title{
Aphelenchoides bicaudatus from Ornamental Nurseries in Taiwan and Its Relationship with Some Agricultural Crops
}

Fan-Yu Jen, Tung-Tsuan Tsay, and Peichen Chen, Department of Plant Pathology, National Chung Hsing University, Taichung, Taiwan, 40223

\begin{abstract}
Jen, F.-Y., Tsay, T.-T., and Chen, P. 2012. Aphelenchoides bicaudatus from ornamental nurseries in Taiwan and its relationship with some agricultural crops. Plant Dis. 96:1763-1766.

Aphelenchoides bicaudatus nematodes were identified in planting materials and water samples from 35 floral ornamental nurseries in Taiwan. A pure line of the nematode was established from the bulk samples collected, and the nematode was identified as A. bicaudatus on the basis of morphometric data and the $18 \mathrm{~S}$ gene sequence (GU984233). Host range tests were conducted by inoculating A. bicaudatus onto Phalaenopsis spp. strawberry, rice, bird's-nest fern,

of A. besseyi). Symptoms did not develop during the 4 weeks following inoculation. However, after staining, both eggs and nematodes inside plant tissue of Phalaenopsis roots, strawberry leaf buds, and rice sheath indicated that $A$. bicaudatus had established in these hosts. These results revealed a potential new host-parasite relationship of $A$. bicaudatus, and suggest that this nematode may infect these economically important hosts.
\end{abstract} cyclamen, dendrobium, African violet, and saxifrage (all reported hosts
The Aphelenchoides genus is divided into plant-parasitic, mycophagousm and predatory groups of nematodes, according to their type of feeding (5). The habitat of Aphelenchoides spp. includes fresh water, soil, insects, plants, and marine water (4), and species are distributed worldwide. In spite of the number of species in the genus, only a few economically important species have been described in detail (9). Within the plant-parasitic group, four species are well studied and recognized: Aphelenchoides fragariae Christie 1932, A. rizemabosi Steiner and Buhrer 1932, A. besseyi Christie 1942, and A. arachidis Bos 1977. Three of these have very wide plant host ranges: A. fragariae colonizes hosts in 47 families and more than 250 species, A. ritzemabosi colonizes plants in 40 families and nearly 200 species, and $A$. besseyi colonizes more than 200 plant species (14).

The most common and economically important Aphelenchoides spp. in Taiwan is A. besseyi $(15,16,18,21)$. A. besseyi parasitizes rice (Oryza sativa L.), strawberry (Fragaria glandiglora Ehrn.), bird's nest fern (Asplenium nidus L.), and many other ornamental plants $(17,18,21)$. A rice field infested with the nematode incurred yield losses of 35 to $45 \%$ (15). In Taiwan, Aphelenchoides besseyi is a common pest of the bird's-nest fern, a cultivated perennial vegetable crop (21). Other Aphelenchoides spp. reported in Taiwan include A. composticola on the button mushroom (Agaricus bisporus (Lange) Imbach) and an unidentified species found on Hydrilla verticillata (L. f.) Royle (20).

Aphelenchoides spp. are also commonly found in the ornamental plants and nurseries, causing significant plant damage $(6,8)$. Aphelenchoides fragariae is able to overwinter in soil, dry infected leaves, and dormant plant crowns (6). In order to establish a standard procedure for producing healthy seedlings, 48 floral ornamen-

Corresponding author: P. Chen, E-mail: janetchen@nchu.edu.tw

Current address of F.-Y. Jen: Taiwan Nihon Nohyaku Co., LTD. Room 902, No. 22, Nanking W. Rd. Taipei, Taiwan, 10352.

* The $e$-Xtra logo stands for "electronic extra" and indicates that a supplementary figure is available online.

Accepted for publication 18 June 2012.

http://dx.doi.org/10.1094/PDIS-03-12-0229-RE

(C) 2012 The American Phytopathological Society tal plant nurseries in Taiwan were surveyed for Aphelenchoides spp. in 2007. The objectives of this study were to (i) investigate the prevalence of soil-inhabiting Aphelenchoides spp. in floral plant nurseries, (ii) identify species of Aphelenchoides found in nurseries based on their morphological characteristics and 18S rDNA sequences, and (iii) evaluate the disease potential of the predominant nematode species on several economically important crops.

\section{Materials and Methods}

Nematode samples from nurseries. A total of 48 nurseries was surveyed: 17, 30, and 1 in central, southern, and eastern Taiwan, respectively. From each nursery, $500 \mathrm{~g}$ of planting medium from 10 to12 pots was collected. The planting medium, herein referred to as "used" planting medium, was mixed well in a bucket. One liter of irrigation water was also collected from each nursery. Nematodes were isolated from (i) three 100-g subsamples of used potting medium, (ii) two 100-g subsamples of potting medium that had not been used, and (iii) all irrigation water samples using a modified Baermann funnel nematode-extraction method (19). Each sample was slowly poured through a double layer of Kleenex tissue placed over a mesh on top of a funnel containing water, and left for $24 \mathrm{~h}$ to allow nematodes to descend into a vial attached to the bottom of the funnel. Samples were observed under a dissection microscope, and those with Aphelenchoides nematodes were recorded.

Establishing a pure-line nematode. Because the number of nematodes in each sample was very small, all Aphelenchoides nematodes in samples obtained from the same region were pooled and transferred onto slant cultures of Alternaria citri on potato dextrose agar to increase their population for further identification, as previously described (18). After 2 weeks, the increased population was collected and observed through a dissection microscope. Based on body size and the position of the vulva, a single female was picked to establish a pure-culture line. The morphological data and the rDNA information included here were from one of the pure lines established.

Morphological identification. The female nematodes were heat-killed and fixed in $2 \%$ triethanolamine (TAF) (7). Specimens were photographed under $\times 40, \times 200, \times 400$, and $\times 1,000 \mathrm{mag}$ nification, and the photographs were calibrated and measured using SPOT software (Diagnostic Instruments). Fifty female nematodes were measured according to de Man's formula, and the data are presented as mean \pm standard deviation. Fine structures were photographed using a cryo-field emission scanning electron micro- 
scope (SEM; JEOL6330 Cryo-FESEM), including the lateral incisures and the tail morphology (20). In preparation for the SEM, nematodes were washed from the culture slants and the volume of water was reduced. The nematode counts were adjusted to approximately 350 to $100 \mu \mathrm{l}$. Delicate Task Wipers (KimberlyClark) were cut into $5 \mathrm{~mm}^{2}$ and two sheets were stacked together. The nematode suspension $(20 \mu \mathrm{l})$ was dripped onto the stacked sheets and the wiper was transferred to the metal carrier of the SEM, adhered to the carrier by double-sided tape. The sample was fixed in liquid $\mathrm{N}_{2}$ for $90 \mathrm{~s}$ and sent into the vacuum chamber for observation. The images obtained were saved with 1,280 by 1,240 pixel resolution as a BMP file.

$18 S$ rDNA sequences. Nematodes from the culture slants were collected via the modified Baermann's funnel method to eliminate most fungal hyphae and spores (20). Approximately 1,500 nematodes were placed into an Eppendorf tube, and the nematode DNA was extracted using the Tissue \& Cell Genomic DNA Purification Kit (GeneMark), following the procedures provided by the manufacturer. The $18 \mathrm{~S}$ rDNA region was amplified using the Nem_18S_F (5'-CGCGAATRGCTCATTACAACAGC-3') and Nem_18S_R (5'-GGGCGGTATCTGATCGCC-3') primers (4), and the polymerase chain reaction (PCR) mix was completed with 0.5 $\mathrm{U}$ of Taq polymerase (GeneMark), and $2.5 \mu \mathrm{l}$ of $10 \times$ Taq buffer in a $20 \mu$ of reaction mixture (3). The PCR conditions were $94^{\circ} \mathrm{C}$ for $4 \mathrm{~min}$ followed by 30 cycles of $94^{\circ} \mathrm{C}$ for $30 \mathrm{~s}, 54^{\circ} \mathrm{C}$ for $30 \mathrm{~s}$, and then $72^{\circ} \mathrm{C}$ for $1 \mathrm{~min}$, and the reaction ended with a final extension at $72^{\circ} \mathrm{C}$ for $5 \mathrm{~min}$. An aliquot of $2 \mu \mathrm{l}$ of the PCR products were used for ligation using the pOSI-T PCR Cloning Kit (GeneMark). The successfully transformed bacteria clones were sent to Mission Biotech Company for sequencing. A consensus sequence of the $18 \mathrm{~S}$ gene was compared with the $18 \mathrm{~S}$ rDNA sequences from other
Aphelenchoides spp. in National Center for Biotechnology Information (NCBI), including Aphelenchoides saprophilus (FJ040408), A. xylocopae (AB252221), A. ritzmabosi (DQ901554), A. stammeri (AB368535), A. blastophtorus (AY284644), A. fragariae (AJ966475), and A. bicaudatus (AY284643). The sequences were paired using DNASTAR software (DNASTAR Inc.) and the percentages of similarities were obtained.

Potential hosts. Economically important plants and crops used in a host range assay included strawberry ( $F$. glandiglora Ehrn.), bird's nest fern (Asplenium nidus L.), rice (O. sativa L. 'Tainung No. 67'), dendrobium (Dendrobium miyakei Schltr.), cyclamen (Cyclamen persicum Mill.), saxifraga (Saxifraga stolonifera Meer b.), and African violet (Saintpaulia ionantha H.). A phalaenopsis orchid (Phalaenopsis Sogo Yukidian 'V3') was also included in this test, because these plants are often raised in moss, which was also sampled from several nurseries in this study. Nematodes for inoculation were propagated on fungal culture slants as previously described (1). Twenty-five rice seeds were soaked in the distilled water with approximately 1,000 Aphelenchoides bicaudatus nematodes until germination and transferred to pots. The nematode suspension was adjusted to 1,000 nematodes per 300 to $500 \mu \mathrm{l}$ for all inoculations. All the available buds on five plants of strawberry or saxifrage were inoculated by dripping the nematode suspension into the buds. For bird's nest fern, dendrobium, cyclamen, and African violet, three leaves per plant were randomly chosen, and the nematode suspension was injected into each leaf. A piece of cotton was used to cover each injection site to retain moisture. Nematodes were inoculated into wounds created by a needle puncture in leaves, buds, or roots of on each of 15 Phalaenopsis orchids (7). Tap water included as the control was mock inoculated in the same manner. All the inoculated plants were observed for symptom

Table 1. Survey results of Aphelenchoides spp. from irrigation water, planting medium, and used planting medium in Taiwan

\begin{tabular}{lccc}
\hline & \multicolumn{2}{c}{ Number of nurseries with } & Aphelenchoides spp. per nurseries surveyed in the region \\
\cline { 2 - 4 } Region in Taiwan & Irrigation water & Planting medium & Used planting medium \\
\hline Central & $1 / 17$ & $1 / 17$ & $13 / 17$ \\
Southern & $0 / 30$ & $2 / 30$ & $21 / 30$ \\
Eastern & $0 / 1$ & $0 / 1$ & $1 / 1$ \\
\hline
\end{tabular}
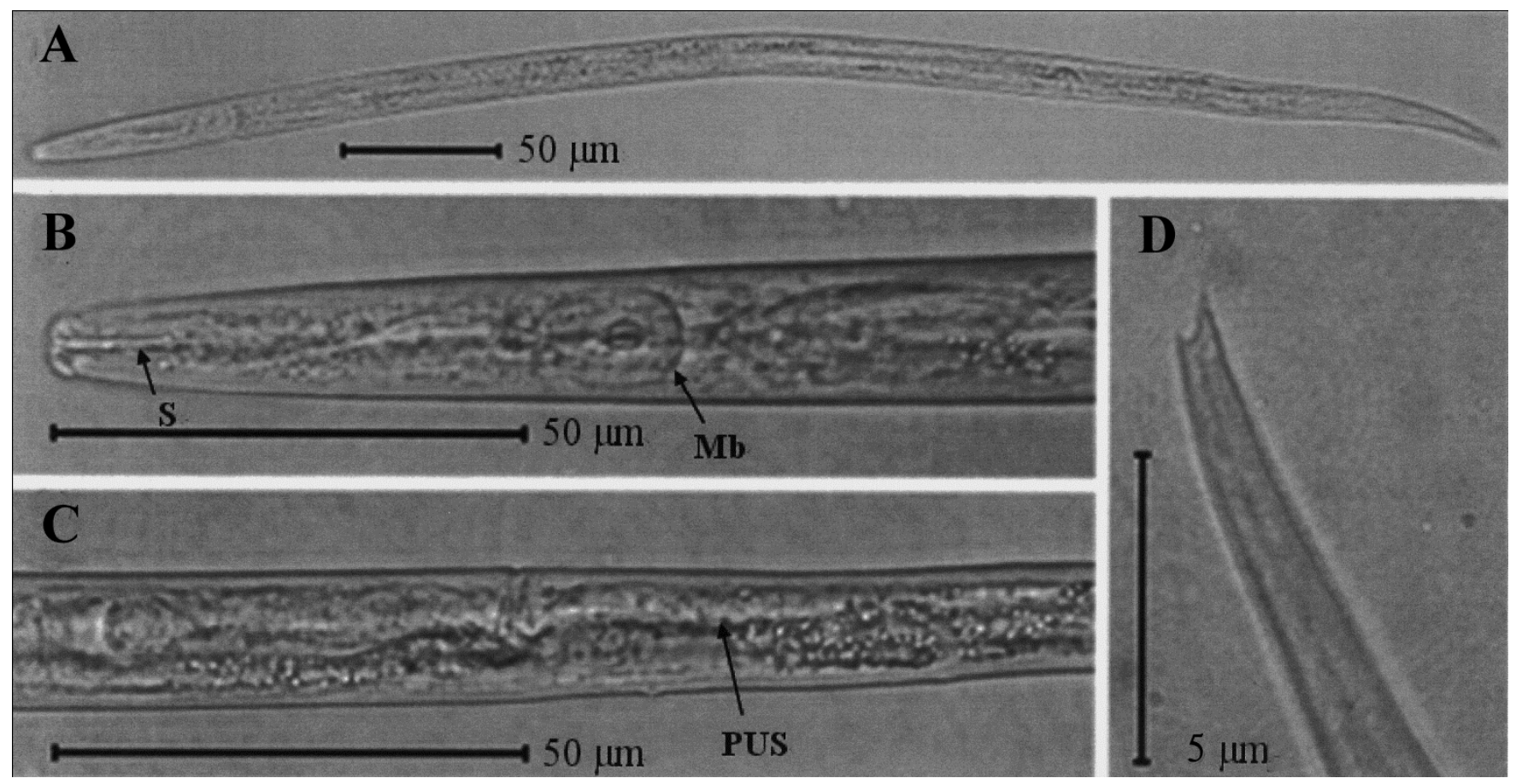

Fig. 1. Photomicrographs of female Aphelenchoides bicaudatus. A, Fixed specimen and B, anterior region; S indicates stylet and Mb indicates middle bulb. C, Posterior region; PUS = post-uterine sac. D, Tail, with typical bifurcation. 
development for 4 weeks, and nematodes from at least one of the replicates in each treatment were reisolated by immersing the inoculation sites in water. The remaining replicates were stained with phenol-cotton blue following the method of Tsay et al. (17). The strawberry, saxifrage, and Phalaenopsis orchid buds were placed in the refrigerator for $1 \mathrm{~min}$ before processing for staining. The inoculated plant tissue was placed in the staining solution and heated in a microwave oven (National, NE-305A) for $30 \mathrm{~s}$ at full power, cooled down for $1 \mathrm{~min}$, and heated again for $30 \mathrm{~s}$ in the microwave oven under the same conditions. To destain the background, the samples were transferred to glycerol and heated for $30 \mathrm{~s}$ before the examination. The host range trials were repeated twice.

\section{Results}

In the 48 nurseries surveyed, no plants exhibited typical symptoms of infection by foliar nematodes. One nursery located in the central region had Aphelenchoides spp. in the irrigation water samples, and three were found to contain Aphelenchoides spp. in planting medium samples (Table 1). Used planting media collected from 35 nurseries were infested with Aphelenchoides spp. (Table 1).

Morphometric data. No male individuals were observed in the pure line cultures. The female of A. bicaudatus is long and slender (Fig. 1A), the head is distinctly set off from the body, appearing smooth, while the lip region is slightly off-set, and the stylet is weak, with stylet knobs (Fig. 1B). The metacorpus is wider than $75 \%$ of the body width (Fig. 1B). The female is prodelphic and the ovary is arranged in a single row; vulva is a transverse slit, twofifths of the body width long and located approximately two-thirds of the body length from the anterior end; the position of vulva from the anterior end, expressed as percentage of body length V\%, was $68.53 \pm 1.2$ with an obvious post-uterine sac (Fig. 1C). The tail tapers gradually beyond the anus, and terminates in a bifurcate tip, of which the ventral prong is acute and always longer than the dorsal prong (Fig. 1D). Under SEM observation, the body has three lateral incisures (Fig. 2).

Fifty females were measured for de Man's formula. Morphometric data, including linear measurements, calculated ratios, and percentage values, were in the range of the paratype of $A$. bicaudatus published by Siddiqui and Taylor (12) (Table 2). Other morphometric data that are important traits for the Aphelenchoides genus are also included, such as the length of the post-uterine sac (18.98 $\pm 4.54)$, presented as the percentage of the length from vulva to anus.

rDNA sequences of $18 \mathrm{~S}$ and internal transcribed spacer regions. A consensus $891 \mathrm{bp}$ of the $18 \mathrm{~S}$ sequence was obtained and submitted to NCBI (GU984233). A BLAST search of the NCBI database exhibited a $99 \%$ similarity with the sequence AY284643, which belonged to A. bicaudatus. The $18 \mathrm{~S}$ sequence in our study

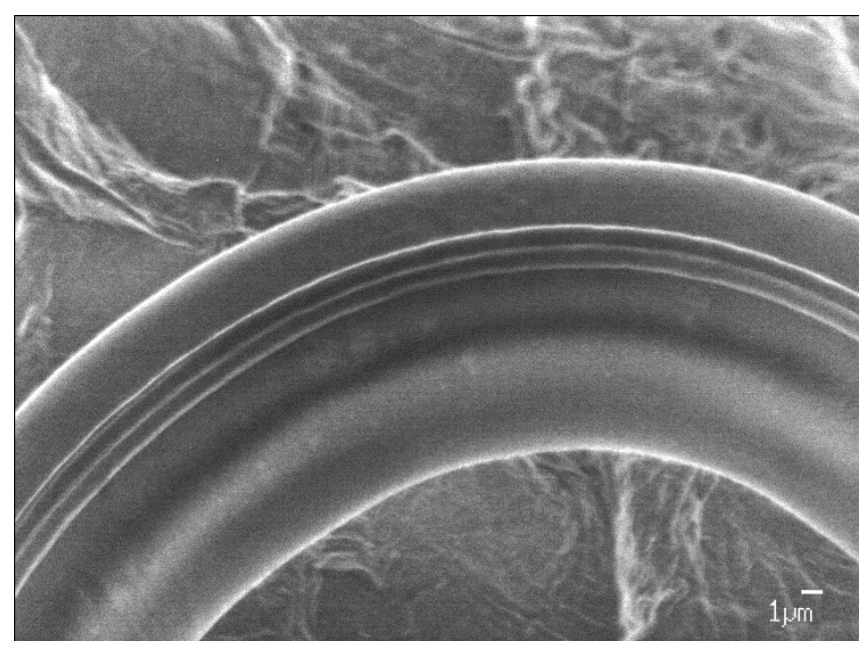

Fig. 2. Three lateral lines of Aphelenchoides bicaudatus observed under cryo-field emission scanning electron microscope $(\times 3,000)$. only had an 81 to $88 \%$ similarity with the other five Aphelenchoides spp. sequences deposited in NCBI. The results confirmed the morphometric data that the specific nematode population obtained from the field samples was A. bicaudatus (Table 3 ).

Potential hosts. Of the eight hosts inoculated with A. bicaudatus, three were apparent hosts of the nematode. After the sheaths of inoculated rice plants were carefully dissected in distilled water, $A$. bicaudatus was observed inside the rice tissue and the nematode was recovered in both trials. Inoculated strawberry plants grew normally but nematodes were reisolated after 4 weeks from 3 of 10 replicates, and were detected inside the buds by staining. The leaves, buds, and roots of Phalaenopsis orchids were inoculated but only the inoculation sites on the roots harbored A. bicaudatus. Staining also revealed eggs inside the orchid root tissue. No nematodes were reisolated from or observed in bird's nest fern, cyclamen, dendrobium, Phalaenopsis leaves and buds, African violet, and saxifrage.

\section{Discussion}

A. fragariae, A. ritzemabosi, and A. besseyi are often reported in ornamental nurseries, and usually cause symptoms in the aerial parts of plants (8). In the current survey, there were no symptoms of infection by foliar nematodes but $A$. bicaudatus was not uncommon. This is the first report of growth and survival of A. bicaudatus inside both rice and Phalaenopsis root tissue, and possibly on strawberry buds. The eggs found inside the Phalaenopsis root tissue suggested that A. bicaudatus was able to colonize and reproduce inside Phalaenopsis roots, although the infection was nonsymptomatic. Many Aphelenchoides spp. are on quarantine lists, and millions of dollars of revenue are lost due to returned

Table 2. Female morphometrics of 50 Aphelenchoides bicaudatus in this study and A. bicaudatus females recorded in 1967

\begin{tabular}{lcc}
\hline & \multicolumn{2}{c}{$\begin{array}{c}\text { A. bicaudatus morphometrics } \\
\text { (mean } \pm \text { standard deviation and range) }\end{array}$} \\
\cline { 2 - 3 } Variable $^{\mathbf{z}}$ & \multicolumn{1}{c}{ This study } & Siddiqui and Taylor (12) \\
\hline$n$ & 50 & 50 \\
$\mathrm{~L}(\mu \mathrm{m})$ & $499.12 \pm 67.95(376-637)$ & $460(410-550)$ \\
$\mathrm{K}(\mu \mathrm{m})$ & $15.24 \pm 2.69(11-22)$ & $\ldots$ \\
$\mathrm{a}$ & $33.03 \pm 2.42(27.00-38.64)$ & $28(25-31)$ \\
$\mathrm{b}$ & $9.0 \pm 0.7(7.5-10.0)$ & $8.2(7.3-9.6)$ \\
$\mathrm{b}^{\prime}$ & $5.13 \pm 0.76(3.61-7.94)$ & $\ldots$ \\
$\mathrm{c}$ & $11.94 \pm 0.93(10.16-14.80)$ & $11.4(9.8-13.7)$ \\
$\mathrm{c}^{\prime}$ & $5.41 \pm 0.56(4.13-7.14)$ & $\ldots$ \\
$\mathrm{V}(\%)$ & $68.53 \pm 1.20(64.90-71.83)$ & $67.5(65-70)$ \\
Stylet $(\mu \mathrm{m})$ & $10.38 \pm 0.63(9-12)$ & $11.2(10-12)$ \\
PUS/V-A $(\%)$ & $18.98 \pm 4.54(9.23-33.80)$ & $\ldots$ \\
\hline
\end{tabular}

${ }^{\mathrm{z}}$ Symbols: $n=$ number of nematodes observed, $\mathrm{L}=$ total body length, $\mathrm{K}=$ maximum body width, $\mathrm{a}=$ total body length divided by maximum body width, $b=$ total body length divided by esophageal length, $b^{\prime}=$ total body length divided by distance from anterior end of body to posterior end of esophageal glands, $\mathrm{c}=$ total body length divided by tail length, $\mathrm{c}^{\prime}=$ tail length divided tail width, $\mathrm{V}(\%)=$ position of vulva from anterior end expressed as percentage of body length, Stylet = length of stylet, and PUS/V-A $(\%)=$ length of post-uterine sac expressed as percentage of length from vulva to anus.

Table 3. Similarities of the 890-bp 18S rDNA sequence from Aphelenchoides bicaudatus isolated in this study compared with six Aphelenchoides spp. sequences obtained from National Center for Biotechnology Information

\begin{tabular}{lcccc}
\hline $\begin{array}{l}\text { Nematode } \\
\text { species }\end{array}$ & $\begin{array}{c}\text { Accession } \\
\text { number }\end{array}$ & Coverage & $\begin{array}{c}\text { Similarities } \\
(\mathbf{\%})\end{array}$ & $\begin{array}{c}\text { Gap } \\
(\mathbf{\%})\end{array}$ \\
\hline A. bicaudatus & AY284643 & $883 / 890$ & 99 & 0 \\
A. fragariae & AJ966475 & $794 / 897$ & 88 & 0 \\
A. blastophtorus & AY284644 & $787 / 901$ & 87 & 1 \\
A. saprophilus & FJ040408 & $785 / 904$ & 86 & 2 \\
A. ritzemabosi & DQ901554 & $746 / 919$ & 81 & 4 \\
A. besseyi & AY508035 & $747 / 919$ & 81 & 3 \\
\hline
\end{tabular}


shipments of nematode-infected plants (6). Further studies needs to be done to clarify the etiology of A. bicaudatus on the Phalaenopsis orchid. A. bicaudatus has been found mostly in soil associated with corn, cotton, potato, sugarcane, rice, and tomato, and is considered primarily a mycophagous nematode $(11,12)$. The nematode is known to reproduce on a number of fungi, algae, yeasts, and Lucerne sprouts $(11,13)$, and has not been reported to parasitize on any economically important hosts.

In the survey, Aphelenchoides spp. were commonly found in used planting media collected from nurseries and, surprisingly, from three batches of planting media that had not been used. It is still not known how these nematodes were introduced into the nurseries. The superfamily Aphelenchoidea has adapted to a wide range of ecological relationships including phytoparasitism, predation, and fungus feeding (2), and many members are able to survive in low-moisture conditions for extensive periods of time (10). The majority of planting media in this survey were moss. Nurseries in Taiwan usually treat the dry commercial moss in a warm water bath for disinfection before use. The treatment apparently is not effective, probably because, when treating large quantities of material, the center of the mass may not reach the desired temperature. Because A. bicaudatus found in this survey might survive in rice, strawberry, and Phalaenopsis orchids, alternative methods of eliminating nematodes in the planting medium are currently under investigation.

When resampling the nursery in which Aphelenchoides nematodes were found in the irrigation water, the predominant nematode was also A. bicaudatus. Ground water is widely used for agricultural irrigation in areas where rainfall is abundant but is not evenly distributed. Therefore, ground water is often stored in uncovered reservoirs without further treatment. To avoid introducing nematodes, simple filtration may remove unwanted nematodes and their eggs.

\section{Acknowledgments}

This work was supported by the Bureau of Animal and Plant Health Inspection and Quarantine Council of Agriculture, Executive Yuan, project numbers: 97-AS-14.4.1-BQ-B2 and 98-AS-9.4.1-BQ-B2.

\section{Literature Cited}

1. Chen, P., and Tsay, T. T. 2006. Effect of crop rotation on Meloidogyne spp. and Pratylenchus spp. populations in strawberry fields in Taiwan. J. Nematol. $38: 339-344$.

2. Cuc, N. T. T., and Pilon, M. 2007. An Aphelenchoides sp. nematode parasitic of Polianthes tuberose in the Mekong Delta. J. Nematol. 39:248-257.

3. Floyd, R. M., Rogers, A. D., Lambshead, P. J. D., and Smith, C. R. 2005. Nematode-specific PCR primers for the 18S small subunit rRNA gene. Mol.
Ecol. Notes 5:611-612.

4. Hunt, D. J. 1993. Aphelenchida, Longidoridae and Trichodoridae: Their Systematics and Bionomics. CAB International, Wallingford, UK.

5. Husain, S. I., and Khan, A. M. 1967. On the status of the genera of the superfamily Aphelenchoidea (Fuchs, 1937) Thorne, 1949 with the descriptions of six new species of nematodes from India. Proc. Helm. Soc. Wash. $34: 167-174$.

6. Jagdale, G. B., and Grewal, P. S. 2006. Infection behavior and overwintering survival of foliar nematodes, Aphelenchoides fragariae, on hosta. J. Nematol. 38:130-136.

7. Lin, Y. Y., Wang, K. M., and Tsay, T. T. 1992. The occurrence of Aphelenchoides besseyi Christie on Dendrobium Lady Fay. Plant Prot. Bull. 34:202215.

8. McCuiston, J. L., Hudson, L. C., Subbotin, S. A., Davis, E. L., and Warfield, C. Y. 2007. Conventional and PCR detection of Aphelenchoides fragariae in diverse ornamental host plant species. J. Nematol. 39:343-355.

9. Nickle, W. R., and Hooper, D. J. 1991. The Aphelenchina: Bud, Leaf, and Insect Nematodes. Pages 465-507 in: Manual of Agricultural Nematology. W. R. Nickle, ed. Marcel Dekker, New York.

10. Otsubo, R., Yoshiga, T., Kondo, E., and Ishibashi, N. 2006. Coiling is not essential to anhydorbiosis by Aphelenchus avenae on agar amended with sucrose. J. Nematol. 38:41-45.

11. Siddiqui, I. A. 1976. Aphelenchoides bicaudatus. CIH Description of PlantParasitic Nematodes, Set 6, No. 84. Commonwealth Institute of Helminthology, St. Albans, UK.

12. Siddiqui, I. A., and Taylor, D. P. 1967. A redescription of Aphelenchoides bicaudatus (Imamura, 1931) Filipjev \& Schuurmans Stekhoven, 1941 (Nematoda: Aphelenchoididae), with a description of the previously undescribed male. Nematologica 13:581-585.

13. Siddiqui, I. A., and Taylor, D. P. 1969. Feeding mechanisms of Aphelenchoides bicaudatus on three fungi and an alga. Nematologica 15:503-509.

14. Tsay, T. T. 1995. Quarantine of plant-parasitic nematodes. Plant Pathol. Bull. 4:43-59.

15. Tsay, T. T., Chen, Y. H., Teng, Y. C., Lee, M. D., Wu, W. S., and Lin, Y. Y. 1998. Bionomics and control of rice white tip disease nematode, Aphelenchoides besseyi. Plant Prot. Bull. 40:277-286.

16. Tsay, T. T., and Cheng, Y. H. 1994. Nematode diseases of flower and ornamental plants in Taiwan. Pages 225-241 in: Special Issue of Taiwan's Flower Pests and Diseases Conference. Taiwan Agricultural Research Institute, Council of Agriculture, Executive Yuan, Taipei, R.O.C.

17. Tsay, T. T., Cheng, Y. H., Chen, H. Y., Lin, Y. Y., and Wu, W. S. 1995 Occurrence and control of nematode diseases on bulbous flowers. Plant Pathol. Bull. 4:180-192.

18. Wang, K. M., Tsay, T. T., and Lin, Y. Y. 1993. The occurrence of Aphelenchoides besseyi on strawberry and its ecology in Taiwan. Plant Prot. Bull. 35:14-29.

19. Wu, H. C., Chen, P. C., and Tsay, T. T. 2010. Assessment of nematode community structure as a bioindicator in river monitoring. Environ. Pollut. 158:1741-1747.

20. Yang, T. H. 2005. The classification and ecology of Aphelenchoides sp. isolated from Hydrilla verticillata. Master's thesis, National Chung Hsing University, Taichung, Taiwan.

21. Yu, P. C., and Tsay, T. T. 2004. Occurrence of a foliar nematode disease of fern in Taiwan. Plant Pathol. Bull. 13:35-44. 